\title{
Evolución de la liquidez monetaria en el Ecuador: años 2000 y 2016
}

\author{
Gladys Urgilés- Urgilés', Karen Chávez- Urgilés²
}

Fecha de recepción: 1 de Febrero 2018

\section{Resumen}

Ecuador perdió la soberanía de desarrollar una política monetaria integral a partir de la dolarización, el presente artículo analiza esta evolución en el país entre los años 2000 y 2016 para lo cual se toma como base la Política Monetaria Moderna y Keynesiana, analizando las condiciones que llevaron a la adopción de la dolarización la inflación, desempleo y caída de los precios del Petróleo. Se analiza los instrumentos monetarios para el encaje legal el mismo que al disminuir hace que los bancos dispongan de mayor dinero para ser puesto en circulación, el dinero electrónico surge con poca fuerza y no tiene confianza en la población, además al tener un Banco Central que no tiene la potestad para emitir moneda, teóricamente la política monetaria se ve anulada pero Carrasco (2016, p. 15) señala el manejo de la estabilidad monetaria no se limita únicamente a la creación de la misma, sino también al control de otros instrumentos necesarios para lograr la liquidez de la economía nacional.

Palabras clave: Liquidez monetaria, dolarización, encaje legal, dinero electrónico.

\section{Abstract}

Ecuador lost its sovereignty to develop an integral monetary policy due to dollarization. This article analyzes this evolution in the country between 2000 and 2006, for which the Modern Monetary and Keynesian Policy have been considered as basis; analyzing the conditions that led to the adoption of dollarization including inflation, unemployment and falling oil prices. An analysis is made of the monetary instruments like the legal reserve which when it decreases, the banks have more money to be put into circulation. Electronic money emerges with little force and has no confidence in the population.

In addition, to not having a Central Bank that can have the power to issue currency, theoretically monetary policy is canceled. According to Carrasco $(2016$, p.15) the management of monetary stability is not limited only to the creation of the same, but also to the control of other instruments necessary to achieve the liquidity of the national economy.

Keywords: Monetary liquidity, dollarization, legal Lace, electronic money.

\section{Introducción}

El estudio de la liquidez en el Ecuador ha brindado la oportunidad a varias investigaciones, Acosta (2003) analizó el nivel de concentración de los depósitos que se generaron en el sistema bancario entre los años 2000 a 2002 observando el posible impacto en la liquidez. El autor observó cómo, en los primeros años de la dolarización, el sector bancario era un factor clave en la búsqueda de la estabilidad económica y la liquidez, por lo cual, si bien el país no cuenta con la posibilidad de desarrollar una política monetaria integral, puede incidir en el sistema bancario para intentar regular la circulación de especies monetarias.

Vera (2007) realizó un estudio titulado "Medición del circulante en Dolarización: Ecuador 2000-2007", en el cual se analizó la distribución de las especias monetarias para proponer dos metodologías para medir el circulante en el país, pues al sustituir el sucre por el dólar dificultó esta operación. Como resultado, el autor pudo determinar los datos de liquidez entre el periodo 2000-2007. Arévalo y Solano (2013) analizaron las consecuencias de elevar el impuesto a la salida de divisas como medida para mantener y aumentar la liquidez en el Ecuador. Los autores pudieron demostrar que esta medida no tuvo una relación directa con los cambios en la liquidez en el país, por lo que no pudo ser una medida regulatoria, y se volvió en cambio, un impuesto recaudador.
Fecha de aceptación: 28 de Marzo 2018

En la década de los noventa la economía del Ecuador se vio envuelta en una serie de situaciones que desembocaron en la crisis económica de 1999 y la implementación de la dolarización (Rojas J., 2015) a partir de lo cual, Ecuador perdió su moneda propia, el sucre, pero adquiría una dinámica diferente, en la que dependería de la valoración de una moneda ajena. La falta de soberanía sobre la política monetaria afectaría al tipo de instrumentos que se podrían aplicar para mantener la estabilidad de la economía y la liquidez ante las variaciones en el ingreso y egreso de divisas. Durante los primeros años de la dolarización se produjo el intercambio de sucres por dólares, pero adicionalmente, el Banco Central del Ecuador (BCE) introdujo una gran parte de la Reserva Monetaria Internacional (RMI) para inyectar divisas al país. A esto deben sumarse los ingresos petroleros y las remesas de migrantes, que favorecieron el aumento de liquidez en el país, señala Larrea (2004, p. 49), al mencionar que "la remesa de divisas, comparable con los ingresos petroleros, permite una recuperación del consumo de los hogares pobres", y Acosta, López y Villama (2005, p. 229), sugieren: "Hay que destacar dos recursos que le dotan de liquidez [a la economía del país]: el petróleo, este momento con precios altos, y las remesas de los emigrantes, que constituyen el segundo rubro de ingresos nacionales".

Para el año 2008, la implementación de una nueva constitución y un nuevo gobierno caracterizado por una política gubernamental que promovía la inversión pública, pudo mantener la liquidez en la economía mediante los ingresos petroleros al alza, aumento en la recaudación de impuestos y los fondos recaudados por el Fondo de Estabilización, Inversión y Reducción del Endeudamiento Público (FEIREP) desde el año 2002, a más de la Cuenta Especial de Reactivación Productiva y Social (CEREPS) y Fondo Ecuatoriano de Inversión en los sectores energético e hidrocarburífero (FEISEH), como sugiere Martin-Mayoral (2009, p. 134), lo que aportó una gran cantidad de recursos al gobierno de turno en la cuenta única del Tesoro. Adicionalmente, las políticas fiscales de corte proteccionista impulsaron una balanza de exportaciones positiva, por ende, mayor ingreso de dólares. Sin embargo, la caída en los precios del petróleo en el año 2015, sumada a un endeudamiento acumulado del Estado hizo evidente la falencia de no poder contar con una política monetaria propia.

Se toman como bases teóricas ciertos aspectos de la Teoría Monetaria Moderna y la Teoría Keynesiana, la Teoría Moderna Monetaria apoya la soberanía de un Estado en su capacidad para producir especies monetarias de cualquier tipo o denominación, al tener siempre la capacidad de producir moneda para cumplir sus compromisos (Huerta, y otros, 2015), por lo mismo, la capacidad para resolver situaciones de liquidez se ve coartada en países que no poseen moneda propia. En el caso de la teoría Keynesiana se considera la manera en que esta promueve la capacidad de un Estado para enfrentarse a los ciclos económicos de crisis o recesión económica a través de políticas anti cíclicas, pero también toman importancia los conceptos de reserva líquida como la cantidad de dinero que no se encuentra en calidad de ahorro, pero tampoco es utilizado transaccionalmente (Piégay \& Rochon, 2006).

La Liquidez puede ser entendida como "la capacidad puntual de convertir los activos en capital líquido o de obtener el capital suficiente para hacer frente a los vencimientos de corto plazo" (Martínez, 2010, p. 16), mientras que para Moya, Brun y Larraga (2010, p. 85) el concepto de liquidez está relacionado con la interacción dada para transformar activos en dinero o viceversa, con el máximo volumen efectivo en el menor plazo. Para un país la liquidez está dada por la cantidad de moneda que circula en el ámbito nacional y por los recursos y/o

Msc. en Administración de Empresas, Docente investigador. Facultad de Industrias Agropecuarias y Ciencias Ambientales en la Universidad Politécnica Estatal del Carchi. Tulcán-Ecuador. e-mail: gladys.urgiles@upec.edu.ec 
reservas de las que puede disponer de ser necesario. Cuando estos recursos disminuyen se produce un riesgo de liquidez, pues a pesar de que puedan existir bienes, productos o depósitos que respaldan un valor, no existen las especies monetarias para facilitar las transacciones necesarias en cualquier sistema económico.

En este sentido, un riesgo de liquidez se produce cuando existen "desajustes entre el exigible y la disponibilidad de fondos para un mismo periodo" (Blanco, 2015, p. 236), lo que puede suceder cuando existe un aumento en las importaciones frente a las exportaciones o disminuye la inversión extranjera o la banca privada coloca un alto porcentaje de fondos en el exterior, entre otras razones. Como resultado, la economía se retrae puesto que existe menos circulante para que la población pueda efectuar las actividades de compra venta de bienes; así mismo, se puede producir un cambio en el ciclo económico e ingresar a una recesión.

Con lo manifestado anteriormente se puede decir que el análisis realizado es de suma importancia puesto que los objetivos orientados al crecimiento y la estabilidad de la economía del país, durante el proceso de la variabilidad de la liquidez en los últimos 16 años permitiendo que el gobierno pueda tomar decisiones inmediatas de mediano o largo plazo para el mejoramiento y la estabilidad económica de todo un pueblo.

\section{Metodología}

El presente artículo abarca un análisis sobre la evolución de la liquidez en el Ecuador entre los años 2000 y 2016, y los instrumentos de política monetaria que se aplicaron para el manejo del dólar, sobre todo, en la manera en que se ha afrontado el reciente periodo. Para esto se realizó un estudio de tipo exploratorio, y también cabe mencionar la estadística descriptiva como método de análisis de la información cuantitativa. El método aplicado para este estudio es el de análisis síntesis. Las técnicas de estudio que se desarrollaron fueron el método bibliográfico documental, fuentes secundarias, información de repositorios digitales, bases estadísticas, publicaciones, análisis de contenidos, análisis personal, análisis crítico a la información estadística obtenida, ley de dolarización, documentos y reglamentos del Banco Central del Ecuador, artículos académicos y bibliografía recopilada. El análisis se efectuó para establecer las posibles causas en las variaciones de la liquidez, y los efectos que pudieron o no tener las medidas ejecutadas por el Estado.

\section{Resultados}

A finales de los años noventa el Ecuador alcanzaba índices históricos en inflación con 43,4\% a diciembre de 1998 y 60,7\% a diciembre de 1999 mientras que el Producto Interno Bruto (PIB) tuvo una tasa de crecimiento negativa mantenida por varios años según el Banco Central del Ecuador (BCE) (2000). Esto era el resultado de la crisis bancaria y económica, que de acuerdo con Barrera, Gallegos y Rodríguez (1999, p. 88), Rojas (2014) se producía por una conjunción de múltiples factores, como el bajo precio del barril de petróleo, la tensión en la frontera con Perú, la Ley de la Agencia de Garantía de Depósitos, el llamado "feriado bancario", el congelamiento de depósitos, el cierre de bancos, la reintroducción del impuesto a la renta, el 1\% a la circulación de capitales, el aumento en los precios de la gasolina y del gas, entre otros. Estos factores fueron elementos que impactaron de forma negativa en la economía del Ecuador y generaron desconfianza en la población del país, como también en la inversión extranjera, sobre todo porque la inflación se disparó a niveles alarmantes. En este escenario la dolarización se planteó como medida para enfrentar la hiperinflación y la devaluación del sucre frente al dólar, sin embargo, en el primer año de dolarización la inflación seguiría en aumento, cerrando el año 2000 en $96 \%$, pero reduciéndose desde entonces hasta llegar a finales del año 2003 el 7,9\%, es decir una cifra de un dígito no vista en varios años. En los años posteriores el valor se mantendría, en promedio, cerca del 3,6\% lo que significa que el país estaría en una situación de estabilización.
La implementación de la dolarización trajo consigo otro panorama para el manejo monetario interno, sobre todo, el rol del BCE. Casals (1985, p. 26) señalaba que los objetivos del BCE, antes de la dolarización, estaban direccionados a cuatro aspectos: equilibrio externo, estabilidad del poder de compra interno, máxima utilización de los factores productivos y propender al desarrollo de la economía. Para esto, el BCE estaba en la facultad de aplicar las políticas monetarias, financiera, crediticia y cambiaria necesarias. En tal punto cabía abordar la interrogante planteada por Carrasco (2016, p. 14) "¿Se puede ejercer política monetaria en la dolarización?". Al tener un Banco Central que no tiene la potestad para emitir moneda, la política monetaria se vería teóricamente anulada, sin embargo, como el mismo Carrasco (2016, p. 15) señala, el manejo de la estabilidad monetaria no se limita únicamente a la creación de la misma, sino también al control de otros instrumentos necesarios para lograr la liquidez de la economía nacional tales como la normativa que rige al sistema financiero y la gestión de las tasas de interés, el encaje legal, entre otros. El objetivo del BCE se movilizó entonces al "diseño, la gestión, el monitoreo y la prospección de las condiciones que garanticen la sustentabilidad" de este entorno dolarizado" (Rojas J., 2015, p. 16). Mediante esta concepción el BCE se mantendría en una postura reguladora y de fortalecimiento a la economía dolarizada, por lo que se puede considerar un acierto ya que fue esta entidad la que gestionó el cambio de moneda, la inyección de circulante, la emisión de moneda fraccionaria, entre otras acciones de control y sostenimiento económico.

Vera (2007, p. 147) expone que, para diciembre de 1999, el BCE adicionó USD 540 millones a un circulante de USD 544 millones, con lo cual el país inició el año 2000 con cerca de USD 1.100 millones en liquidez y cerró ese mismo año en más de USD 4.000 millones. En años posteriores se mantuvo una tendencia creciente en la oferta monetaria, constituida por las especies en circulación, la moneda fraccionaria importada junto con la emitida por el BCE y los depósitos a la vista lo que tuvo un efecto positivo en la liquidez y movilizó la economía.

En la figura 1 se observa cómo la liquidez ha ido constantemente en aumento, iniciando con un valor de USD 4.875 millones a diciembre del año 2000, y llegando a USD 4.618 millones para diciembre del 2016. Este aumento es positivo para el país, el valor del circulante que puede ser causado por los ingresos por remesas y la venta de petróleo, sobre todo en el periodo entre el 2003 y el 2008, en el cual existió una variación de USD 7.667 millones a USD 17.461 millones. Entre el año 2008 y el año 2009 el crecimiento es menor, pasando de USD 17.461 millones a USD 18.831, y se trató de un periodo de poca estabilidad política que se reflejó en una reducción de la inversión extranjera y la apertura crediticia de los bancos, pues el riesgo país se elevó a 4.731 puntos para diciembre del 2008 (Banco Central del Ecuador, 2017). Desde el 2009 en adelante la liquidez volvería a crecer de manera importante pues el gobierno inyectó una gran cantidad de circulante en la economía mediante la inversión en obras públicas, la entrega de bonos, en enfoque de economía popular y solidaria, entre otras acciones respaldadas en la gran cantidad de recursos inyectados a la Cuenta Única del Tesoro por la eliminación de fondos petroleros y la valorización del precio del petróleo. Para el año 2014 la liquidez alcanzó un valor de USD 40.104 millones en contraste con USD 1.831 millones para el año 2009

La inyección de liquidez por parte del Estado tuvo sus beneficios, pero también desventajas. Al contar con una gran cantidad de presupuesto desde el año 2009. Esta época de bonanza se reflejó en una disminución del desempleo y en mayor poder adquisitivo, como también en una mayor apertura crediticia en las instituciones bancarias, como resultado la liquidez aumentó de manera importante. La desventaja reside en que, la falta de una política de austeridad, que considere el ahorro como una reserva futura, impidió que pueda sobrellevarse de mejor manera la caída en los ingresos petroleros en el año 2014. Entre el año 2015 y 2016 se intentó mitigar la crisis de insolvencia mediante la inyección de capital desde el Estado, y mediante créditos exteriores 
lo que puede tener un efecto similar al mencionado, es decir, un aumento de la liquidez al corto plazo, pero un decrecimiento de la misma al mediano y largo plazo.

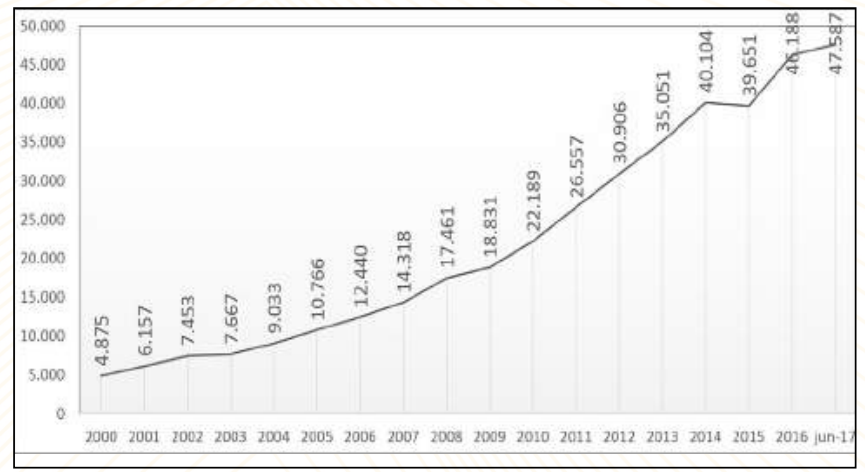

Fuente: (Banco Central del Ecuador, 2016)

Figura 1. Liquidez en millones de dólares

En la figura 2 se presenta el crecimiento de la liquidez por cada año, respecto al periodo anterior durante los años que corresponde la liquidez del año 2000 en relación con la liquidez de 1999. Las variaciones se produjeron debido a que parte de las reservas internacionales en forma de dólar se trajeron como circulante al Ecuador, siendo necesario que exista gran cantidad de circulante en dólares para poder hacer una transición rápida entre monedas. A partir de entonces la liquidez se manejaría, mediante la inyección directa de moneda, mediante políticas fiscales, o mediante la aplicación de instrumentos de política monetaria.

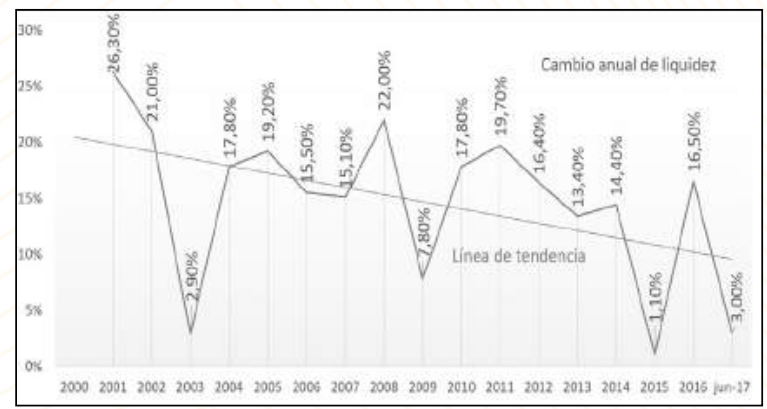

Fuente: (Banco Central del Ecuador, 2016) Figura 2. Fluctuación en la liquidez, \% de crecimiento

En cuanto a los instrumentos de política monetaria aplicados durante el periodo 2000 y 2016, puede mencionarse a aquellos que no dependen directamente de la producción de moneda, como el encaje legal que se mantuvo en aplicación por parte del BCE. Para Baca y Marcelino (2016, p. 12) el encaje legal es entendido como un porcentaje de los depósitos existentes en la banca que deben funcionar como reserva o garantía. En los inicios de la dolarización el encaje legal se encontraba por el 9\%, el mismo que disminuyó hasta el $4 \%$ para el año 2001. Si se disminuye el encaje legal los bancos disponen de mayor dinero, y este a su vez puede ser puesto en circulación al expandir la capacidad crediticia de la entidad, por ejemplo, mediante la entrega de créditos de consumo. Esta medida tuvo un impacto positivo pues en el año 2001 la liquidez aumentó un 21\% con relación al año 2000.

Para el año 2003 se reduce el crecimiento de la liquidez con un porcentaje del 2,9\% con relación al año 2002. En aquel año el BCE envió al exterior USD 680,9 millones en billetes de alta denominación y billetes deteriorados, e importó USD 682,20 millones (BCE, 2003). En este periodo se produce una reducción en el crecimiento de la liquidez por la reposición de una gran cantidad de billetes en mal estado, pues en el país se presume una vida útil de los mismos de entre 3 y 5 años, mientras que en los Estados Unidos el promedio de vida de un billete es de 10 años. Esta reposición de moneda afecta al circulante de baja denominación siendo la que más afectada se ve por el uso frecuente. En este mismo año el encaje legal se reduce al $2 \%$ en un intento por dotar de mayor liquidez a la banca privada. Este encaje legal se mantuvo en el $2 \%$, hasta su elevación al $5 \%$ en noviembre del 2016, situación que afectaría la liquidez de las entidades bancarias.

Con la finalidad de elevar la liquidez para el año 2004, uno de los instrumentos manejados por el Banco Central del Ecuador fue la recirculación de liquidez consistente en "recoger los excedentes de recursos que existen mediante la emisión de Títulos del Banco Central posteriormente redistribuirlos y canalizarlos, a través de Operaciones de Reporto hacia instituciones financieras" (BCE, 2003), de esta manera se pretendía solventar las necesidades de liquidez que pudieran presentar las instituciones del sistema bancario. En otras palabras, cuando las instituciones bancarias tenían necesidades de liquidez, vendían títulos de valores al BCE por un monto especificado y avalados por el gobierno nacional, los cuáles debían ser recomprados por dichas instituciones a la fecha de vencimiento; con esto, las entidades bancarias contaban con fondos disponibles inmediatamente.

Como resultado, se puede considerar que la aplicación de la recirculación de capital apoyó el crecimiento de la tasa de liquidez, que se elevó por encima de los 15 puntos porcentuales al año, llegando al año 2008 con un 22\%, esto significaba pasar de 9.033 millones de dólares en el año 2004 a 17.461 millones de dólares en el 2008. En este periodo existen factores anexos tales como remesas de migrantes en el exterior, y la elevación en los precios del petróleo, que significaron una gran cantidad de divisas ingresadas al país y por tanto un aumento en el circulante.

El gobierno entrante en el 2008, liderado por el economista Rafael Correa, concibió un cambio en la política fiscal y monetaria, optando por la inversión pública en vez de la acumulación de reservas. Existía, a esas fechas el impacto de la crisis internacional, una disminución en la venta y en los precios del petróleo, y la reducción en el crédito por parte del sistema bancario que pretendía asegurar su propia liquidez frente a indicadores de riesgo país que se dispararon a más de 4.000 puntos al cierre del año. Para el 2009 la liquidez fue de 18.831 millones, lo que representaba una caída en la tasa de crecimiento, con un $7,86 \%$ respecto el año 2008.

En el año 2009 se promulga la Ley Orgánica para la Recuperación del Uso Público de los Recursos Petroleros, agregándolos a la Cuenta única del Tesoro del Estado, lo que le aportó una gran cantidad de recursos para la utilización de la inversión pública, puesto que los fondos petroleros ecuatorianos (FEP - FEIREP - CEREPS - FAC - FEISEH) fueron aumentando hasta llegar al año 2007 a un valor de 3.193 millones de dólares (Baquero \& Mieles, 2015). Durante los siguientes años parte de este presupuesto se invertiría en multitud de obras públicas, generando empleos, por lo que estos fondos estatales pasaron a enriquecer la cantidad de especies monetarias en circulación elevando nuevamente la liquidez. Para el año 2010 la liquidez creció en un 17,8\% y se mantuvo por sobre el 13\% anual hasta el año 2014.

En el 2015 el Ecuador afrontaría una nueva crisis lo que afectó su liquidez de manera importante, pues es el único año en que se generó un decrecimiento del $-1,1 \%$. En ese año se produce la caída en los precios del petróleo, la valoración del dólar frente a la moneda de muchos de los socios comerciales del país, y al estar en parte sometidos a la política monetaria estadounidense, se vio afectado el ingreso de divisas. Para el Estado esta situación se traduce en la reducción de su presupuesto, por lo cual recurrió a créditos externos para sobrellevar la situación. La liquidez se redujo, y la economía se contrajo, entrando en estado de recesión. Las medidas adoptadas por la banca privada les permitió mantener una liquidez superior al 30\% (El Telégrafo, 2016) que junto a la inyección de capital mediante préstamos al exterior y la utilización de un porcentaje de las reservas internacionales, reflejó en una liquidez de USD 46.188 millones para el 2016, en comparación de un valor de USD 39.651 millones en el 2015. 
Las demandas de billetes se realizan a partir de las reservas internacionales, lo que representó para el Ecuador cerca de 3,7 millones de dólares en el año 2015 y 2,3 millones de dólares en el año 2016 según el BCE (2015; 2016). Una de las finalidades de manejar una moneda digital equivalente a la moneda en curso, es mantener la liquidez y reducir los gastos monetarios por la transportación de divisas. En teoría, al incrementarse el uso del dinero electrónico sustentado en fondos de la Reserva Internacional de Libre Disponibilidad (RILD), se reduciría la necesidad de transportar dinero físico, eliminando dichos costos.

\section{Conclusiones}

En Ecuador la inflación se disparó a niveles alarmantes como resultado de la crisis bancaria y económica y según Barrera, Gallegos y Rodríguez (1999, p. 88), Rojas (2014) era provocada por el bajo precio del barril de petróleo, la tensión en la frontera con Perú, la Ley de la Agencia de Garantía de Depósitos, el llamado "feriado bancario", el congelamiento de depósitos, el cierre de bancos, la reintroducción del impuesto a la renta, el 1\% a la circulación de capitales, el aumento en los precios de la gasolina y del gas, entre otros.

La dolarización se planteó como medida para enfrentar la hiperinflación y la devaluación del sucre frente al dólar cerrando en el año 2000 con el 96\%, reduciéndose desde entonces hasta al año 2003 con el 7,9\% es decir una cifra no vista en varios años.

La promulgación de la Ley Orgánica para la Recuperación del Uso Público de los Recursos Petroleros agregados a la Cuenta Única del Tesoro del Estado en el 2009, aportó recursos para la utilización de la inversión pública hasta llegar al año 2007 con un valor de 3.193 millones de dólares (Baquero \& Mieles, 2015), en el año 2010 la liquidez creció en un $17,8 \%$ y se mantuvo por sobre el $13 \%$ anual hasta el año 2014 , Ecuador afrontó una nueva crisis en el 2015 lo que afectó su liquidez de manera importante, con un decrecimiento del $-1,1 \%$, con una liquidez de USD 46.188 millones para el 2016.

Las demandas de billetes se realizan a partir de las reservas internacionales, lo que representó para el Ecuador cerca de 3,7 millones de dólares en el año 2015 y 2,3 millones en el año 2016 según el BCE (2015; 2016). El dinero electrónico ha surgido con poca fuerza la moneda digital equivale a la moneda en curso, manteniendo la liquidez y reduciendo los gastos monetarios por la transportación de divisas, al incrementarse el uso del dinero electrónico sustentado en fondos de la Reserva Internacional de Libre Disponibilidad (RILD), se reduciría la necesidad de transportar dinero físico, eliminando dichos costos.

\section{Referencias}

Acosta, A., López, S., \& Villamar, D. (2005). Las remesas y su aporte para la economía. Revista La migración ecuatoriana: transnacionalismo, redes e identidades, 227-252.

Acosta, G. (2003). Concentración bancaria de los depósitos del sector bancario ecuatoriano en el periodo 2000 a 2002. Tesis de Economía, Pontifica Universidad Católica del Ecuador, Facultad de Economía, Quito. Obtenido de http://repositorio.puce.edu.ec/handle/22000/1417

Arevalo, V., \& Solano, K. (2013). Análisis del efecto del alza del impuesto a la salida de divisas en la liquidez de la economía ecuatoriana. Tesis de Grado, Quito, Ecuador. Obtenido de http://www.dspace.espol.edu.ec/ xmlui/handle/123456789/21553

Baca, G., \& Marcelino, M. (2016). Ingeniería Financiera. México: Grupo Editorial Patria.

Banco Central del Ecuador. (2000). Memoria 1999. Quito: Banco Central del Ecuador.

Banco Central del Ecuador. (2003). Memoria 2003. Obtenido de Banco Central del Ecuador: https://contenido.bce.fin.ec/documentos/PublicacionesNotas/Catalogo/Memoria/2003/cap3.pdf

Banco Central del Ecuador. (2015). Estado de Resultados de Enero 1 a Diciembre 30 de 2015. Obtenido de Banco Central del Ecuador: https:// www.bce.fin.ec/images/transparencia/informacion_financiera/documentos/ estadosfinancieros/era2015.pdf

Banco Central del Ecuador. (2016). Estado de Resultados de Enero 1 a Diciembre 30 del 2016. Obtenido de Banco Central del Ecuador: https:// www.bce.fin.ec/images/transparencia/informacion_financiera/documentos/ estadosfinancieros/era2016.pdf

Banco Central del Ecuador. (Junio de 2016). i) Información completa y detallada de los procesos precontractuales, contractuales, de adjudicación y liquidación, de las contrataciones de obras, adquisición de bienes, prestación de servicios, arrendamientos mercantiles, etc.,... Junio 2016. Obtenido de Banco Central del Ecuador: https://www.bce.fin.ec/images/ transparencia2016/literalijunio2016.pdf

Banco Central del Ecuador. (2017). Rlesgo País. Obtenido de Banco Central del Ecuador: https://contenido.bce.fin.ec/resumen_ticker. php?ticker_value=riesgo_pais

Baquero, D., \& Mieles, J. (2015). El 'boom' petrolero y el ahorro que hoy hace falta en Ecuador. Foro Economia Ecuador, 4. Obtenido de http://foroeconomiaecuador.com/fee/el-boom-petrolero-y-el-ahorro-quehoy-hace-falta-en-ecuador/

Barrera, A., Gallegos, F., \& Rodríguez, L. (1999). Ecuador: un modelo para [des]armar. Quito: Editorial Abya Yala.

Blanco, A. (2015). Gestión de Entidades Financieras: Un enfoque práctico de la gestión bancaria actual. Madrid: ESIC Editorial.

Carrasco, A. (Enero de 2016). La Política Monetaria en el Ecuador, con régimen cambiario dolarizado. Revista Fiscalidad(10). Obtenido de Servicio de Rentas Internas: https://cef.sri.gob.ec/pluginfile.php/20139/ mod_page/content/78/RF10_1.pdf

Casals, J. (1985). Objetivos de Política Monetaria y Funciones del Banco Central del Ecuador. Quito: Publicaciones del Banco Central del Ecuador. Obtenido de https://www.bce.fin.ec/cuestiones_economicas/images/PDFS/1985/No11/No.11-1985\%20CasalsJuan.pdf

El Telégrafo. (23 de septiembre de 2016). A 33,2\% llega la liquidez en entidades bancarias privadas. El Telégrafo Edición digital. Obtenido de http://www. eltelegrafo.com.ec/noticias/economia/8/a-33-2-Ilega-la-liquidez-en-entidades-bancarias-privadas

El Telégrafo. (30 de Agosto de 2017). El sistema de dinero electrónico dejará de ser administrado por el Banco Central. Obtenido de El Telégrafo Edición Digital: http://www. eltelegrafo.com.ec/noticias/economia/8/el-sistema-de-dinero-electronico-dejara-de-ser-administrado-por-el-banco-central

Fondo Monetario Internacional. (2013). Reservas Internacionales y Liquidez en Moneda Extranjera. Washington D.C.: International Monetary Fund, Publication Services.

García, R. (2004). Moneda, banca y política monetaria. EUNED.

Huerta, A., Martín, J., Moreno, A., Bortz, P., García, C., \& Wray, R. (2015). Teoría Monetaria Moderna: Manual de macroeconomía sobre los sistemas monetarios soberanos. Lola Books.

Larrea, C. (2004). Dolarización y Desarrollo Humano en Ecuador. Iconos, Revista de Ciencias Sociales, 19, 43-53. Obtenido de http://www. redalyc.org/htm//509/50901906/

Martínez, J. (2010). Manual de análisis financiero. Ediciones digitales.

Martín-Mayoral, F. (2009). Estado y Mercado en la historia de Ecuador: Desde los años 50 hasta el gobierno de Rafael Correa. Revista Nueva Sociedad, 221, 120-137. Obtenido de https://search.proquest.com/ openview/aafObeae $3 f d b b 1$ dadeaa $57 f 43 d 9 f d 4 d 6 / 1$ ?pq-origsite $=g s c h o /-$ $a r \& c b l=13322$

Moya, M., Brun, X., \& Larraga, P. (2010). Cómo interpretar la información económica: análisis de mercados financieros, coyuntura económica, sistema financiero, política monetaria. Barcelona: Profit Editorial.

Piégay, P., \& Rochon, L.-P. (2006). Teorías monetarias poskeynesianas. Madrid: Ediciones AKAL.

Rojas, C. (29 de mayo de 2014). Las 9 claves para entender la crisis de 1999 y la caída de Mahuad. El Comercio. Obtenido de http://www. elcomercio.com/actualidad/mahuad-peculado-feriado-bancario-ecuador. html

Rojas, J. (2015). El proceso de dolarización, balanza de pagos, sector externo, su impacto e incidencia en el sector real de la economía ecuatoriana. Cuenca: Universidad Politécnica Salesiana.

Vera, W. (2007). Medición del circulante en dolarización: Ecuador 2000-2007. Cuestiones Económicas, 23(2), 133-161. Obtenido de https:// contenido.bce.fin.ec/documentos/PublicacionesNotas/Catalogo/Cuestiones/ XXIII-II-04Vera.pdf

\section{Anexo página 25}

